\title{
One-year visual outcome of secondary piggyback pinhole device implantation in pseudophakic eyes with irregular corneal astigmatism and iris trauma
}

\author{
Vivian W. M. Ho ${ }^{1} \cdot$ Mohamed Elalfy $^{1,2} \cdot$ Samer Hamada $^{1} \cdot$ Damian Lake $^{1}$ \\ Received: 28 July 2020 / Revised: 1 March 2021 / Accepted: 6 April 2021 / Published online: 22 April 2021 \\ (c) The Author(s), under exclusive licence to The Royal College of Ophthalmologists 2021
}

\begin{abstract}
Purpose Retrospective case series evaluating the efficacy and safety of implanting XtraFocus ${ }^{\mathrm{TM}}$ pinhole device in pseudophakic patients with irregular corneal astigmatism with concurrent or standalone iris defect.

Methods Electronic case records were searched for: uncorrected distance visual acuities (UDVA) and corrected distance visual acuities (CDVA), automatic or manifest refraction and spherical equivalent (SE). All main outcomes were evaluated preoperatively and postoperatively at 1, 3, 6,9 and 12 months, along with patients' satisfactory outcome and complications. Results Eleven pseudophakic eyes of 11 patients with a mean age of 54 (range 27-81) years were included. Median UDVA improved significantly from $\operatorname{logMAR} 0.7$ (range $0.1-1.22$ ) pre-operatively to 0.4 (range $0-1.3$ ) at 1 -month $(p=0.002$ ); median CDVA remained unchanged at logMAR 0.4 (range 0-0.1) pre-operatively and 0.4 (range 0-0.8) at 1-month $(p=$ 0.36). There were no significant statistical differences in both UDVA and CDVA between the post-operative periods. Ten patients $(90.9 \%)$ had initial UDVA improvement at 1-month post op. Eight $(72.7 \%)$ patients expressed satisfaction with improved vision or reduction of glare/halos. Three (27.3\%) patients had unsatisfactory visual outcome resulting in 2 requested for implant explantation due to worsening of glare and distressing floaters.

Conclusions XtraFocus ${ }^{\mathrm{TM}}$ is effective in improving vision or reducing glare in pseudophakic patients with irregular corneal astigmatism or intragenic iris trauma, with over $70 \%$ expressed satisfactory outcome. Disturbing floaters and glare preclude its use in some resulting in implant explantation.
\end{abstract}

\section{Introduction}

Irregular astigmatism causes blurred vision that is uncorrectable by spectacle glasses. It is commonly seen in patients with keratoconus, corneal dystrophies, iatrogenic procedures such as keratoplasty, photorefractive keratectomy (PRK), laser in situ keratomileusis or corneal scarring secondary to trauma or infection. Treatment aims at rearranging the orientation of the astigmatism so that it is more regular and amenable to sphero-cylindrical correction. Current management options include contact lens wear such as rigid gas-permeable [1], hybrid [2] or scleral

Vivian W. M. Ho

vivian1118@hotmail.com

1 Corneoplastic Unit and Eye Bank, Queen Victoria Hospital, Holtye Rd, East Grinstead, England

2 Cornea Unit, Research Institute of Ophthalmology, Giza, Egypt lens [3], topography-guided laser refractive surgery [4], or intrastromal corneal ring segments (ICRS) [5, 6]. However, there are pros and cons with each of these treatments and may not be suitable for patients with poor ocular surface, those with contact lens intolerance or corneas too thin for the laser refractive surgery or ICRS to be performed safely.

Iris defects could be a result of congenital conditions or secondary from trauma or iatrogenic causes. They could be treated with cosmetic contact lens, surgical pupilloplasty or an artificial iris implant insertion.

XtraFocus $^{\text {TM }}$ pinhole intraocular implant Morcher $^{\circledR}$ $\mathrm{GmbH}$ ), is a foldable hydrophobic acrylic ciliary sulcus lens with no refractive power, received the Conformite Européenne mark in 2016. Its main indication is for the treatment of irregular corneal astigmatism in pseudophakic patients, including keratoconus, pellucid marginal degeneration, post-radial keratotomy, post-penetrating keratoplasty, post-LASIK ectasia and traumatic corneal laceration. Its secondary indications are near vision enhancement in 
monofocal pseudophakia and reduction of dysphotopsias in multifocal pseudophakia. Due to its black opaque diaphragm, it has also been used in patients with combined irregular astigmatism and large iris defect $[7,8]$.

We present our experience from a UK tertiary eye care centre with the use of XtraFocus ${ }^{\mathrm{TM}}$ pinhole device in pseudophakic patients with irregular corneal astigmatism with concurrent or standalone iris defect.

\section{Patients and methods}

This is a retrospective case series. The case notes of all pseudophakic patients who had XtraFocus ${ }^{\mathrm{TM}}$ implantation were reviewed. Patients with any intra-operative complications were excluded. Surgeries were performed by multiple surgeons (Corneal consultants or their fellows) as a single surgical procedure, at the Queen Victoria Hospital, East Grinstead, United Kingdom between May 2018 and Sept 2019. Surgeries were performed under either local or general anaesthesia through a superior $2.75 \mathrm{~mm}$ clear corneal incision with the implant inserted into the ciliary sulcus. Electronic case records were searched for the following outcomes: uncorrected distance visual acuities (UDVA) and corrected distance visual acuities (CDVA) in Snellen fraction, automatic or manifest refraction and spherical equivalent (SE) in dioptres (D). Visual acuity measurements were converted to logarithm of the minimum angle of resolution $(\log$ MAR) notation for statistical analysis. All main outcomes were evaluated preoperatively and postoperatively at 1, 3, 6, 9 and 12 months, with all complications reported. Variables are expressed by their mean, median, minimum and maximum. A $p$ value $<0.05$ was considered statistically significant. All analyses were performed using JASP Team (2020, Version 0.12.2) [Computer software].

\section{Results}

A total of 11 pseudophakic eyes of 11 patients were included. Their mean age was 54 (range 27-81) years with a mean follow-up of 11.6 months (range 4.37-18.57). Mean keratometry was $44.75 \mathrm{D}$ (range 40.5-53) and mean maximal keratometry (Kmax) was 54.15D (range 42.5-75.6). Patients' demographics and characteristics are summarised in Table 1. Pre-operatively presenting complaints included glare (eight patients) and poor vision (three patients). Indications for an XtraFocus ${ }^{\mathrm{TM}}$ implantation were (i) irregular astigmatism secondary from post-keratoplasty (six patients), keratoconus (two patients), corneal scar (one patient) and/ or (ii) iatrogenic iris defect (six patients, four with concurrent irregular astigmatism).

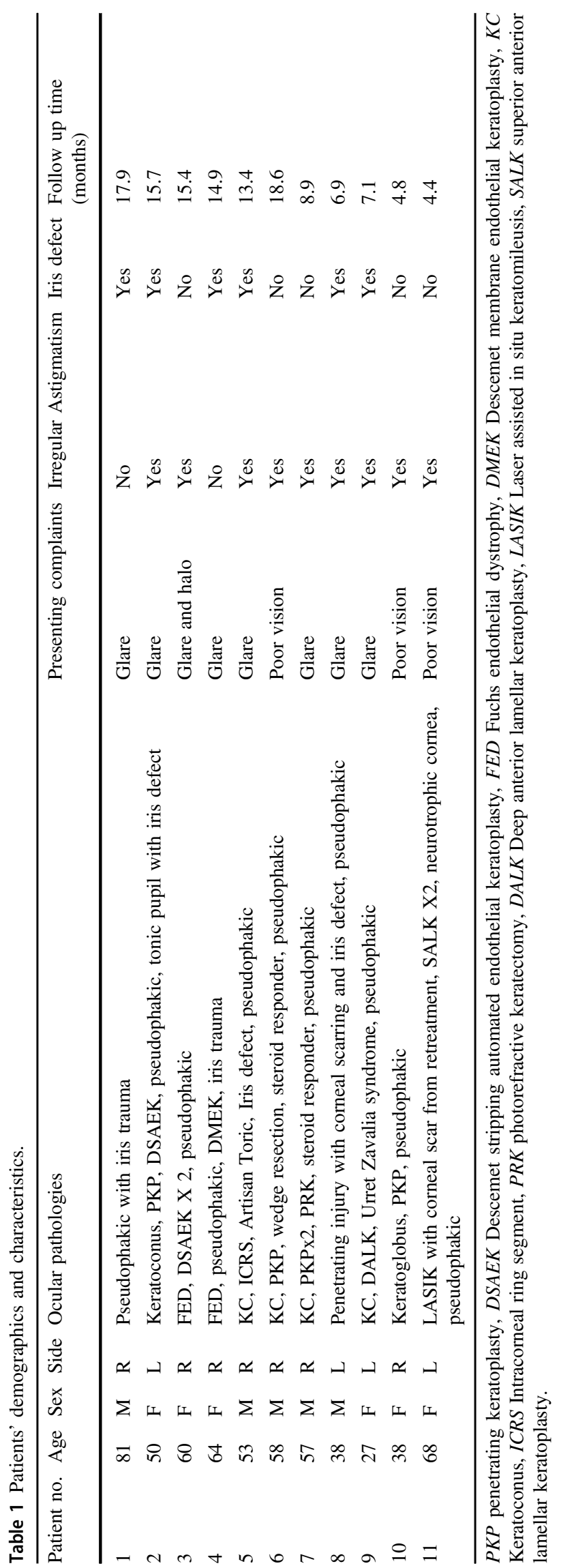




\section{Efficacy}

Mean UDVA improved significantly at 1-month, 6-month and 12-month post-op $(p<0.05)$ and mean CDVA showed no difference. Mean SE changed significantly at 1 -month, 3-month, 9-month and 12-month post-op ( $p<0.05)$.

Ten patients $(90.9 \%)$ had initial UDVA improvement and had either stable or improved CDVA at 1-month post op. Visual acuity remained stable in eight $(72.7 \%)$ patients throughout the follow-up periods who expressed satisfactory outcome with improved vision or reduction of glare/ halos.

Each patients' preoperative and post-operative visual acuity expressed as $\operatorname{logMAR}, \mathrm{SE}$ in D, pre-op Kmax in D, index of surface variance (ISV), satisfaction (yes or no) and complications are shown in Table 2a. Mean UDVA, CDVA and SE preoperatively and up to 12 months postoperatively are shown in Table $2 b$.

\section{Post-operative complications}

Four post-operative complications were reported but only two were directly related to the pinhole implant. Both had initial visual improvement but developed persistent glare or distressing floaters at 3-month and 6-month respectively, resulting in one having implant explantation at 10-month post op with complete resolution of glare; and the other one was waiting for explantation at the time of writing this manuscript.

The other two complications had no direct relation to the pinhole device: one patient developed moderate subluxation of the pinhole device at 1-week despite an uneventful implantation, patient chose to be observed at the time of writing this manuscript. The other patient developed an iris displacement resulting in significant photophobia, requiring iris repair at 3-month with vision improvement and complete resolution of symptoms.

\section{Discussion}

In this retrospective study, we share our experience in the use of the Xtrafocus ${ }^{\mathrm{TM}}$ pinhole device in pseudophakic patients with irregular astigmatism with concurrent or standalone iris defect. Our results showed an initial visual improvement in $90 \%$ of patients and over $70 \%$ remained a stable vision throughout the follow-up periods. These results are comparable to a previous study of Trindade et al. [7], who first reported their series in 2017 of 21 patients with irregular astigmatism and a mean follow up of 22 months. They reported a significant improvement in both UDVA and CDVA at 1-month post op with stable vision in the following months. The authors recommended the device to be used in patients with both irregular astigmatism and concurrent iris defect as they reported greatest visual improvement in these cases. Agarwal et al [8]. also reported a patient with irregular astigmatism due to a corneal scar and iris trauma resulting with glare and photophobia, benefited from the pinhole implant with complete resolution of symptoms and improved uncorrected distance, intermediate and near visual acuity. Our results demonstrated similar findings though only three out of four patients with both pathologies reported satisfactory outcome (described later in text).

There was a significant hyperopic shift in the mean SE post-operatively in our group of patients whereas Trindade et al. [7] reported no change in the manifest refraction in their series. This result should be viewed with caution as majority of our refraction was measured with an autorefractor. Autofraction is known to be unreliable [9] in patients with keratoconus, in addition a reproducible subjective refraction was difficult to perform on our patients due to poor vision or corneal scarring.

Despite the encouraging visual results, there are side effects associated with the pinhole implant. Haloes, poor vision in dim light, floaters and restricted field of vision have been reported [7, 10, 11]. Faint haloes around light source had been reported in two patients [7] and reduced visual acuity under low-light condition in another two patients $[7,10]$. One of them had severe poor vision in dim light despite an initial visual improvement, requiring implant explantation [10]. Agarwal et al. [11] reported one patient noted with a single vitreous condensation preoperatively, complained of troublesome floaters following pinhole implantation, subsequently had explantation at 3 months after surgery. In our study, two patients complained of persistent glare/halos and distressing floaters despite an initial improvement in vision. One patient noticed glare and haloes at 3 months post-op that persisted, requiring explanation at 10 months post op with complete symptoms resolution. The second patient started experiencing distressing floaters and worsening of vision at 6-months post-op, with no floaters noted preoperatively and a normal retinal examination; the patient was waiting for explantation at the time of writing this manuscript. Both implantations were uneventful with a well-centered implant with no post-operative trauma recorded. There was no obvious explanation to their visual symptoms. Floaterectomy may help in the second patient but it carries certain risks [12]. Agarwal et al. [11] believed the pinhole accentuates even small-medium vitreous opacities and creates such visual disturbance. It is therefore important to perform a thorough fundal examination preoperatively and to counsel patients that distressing floaters could occur following the pinhole implant. 
One-year visual outcome of secondary piggyback pinhole device implantation in pseudophakic eyes with...

815

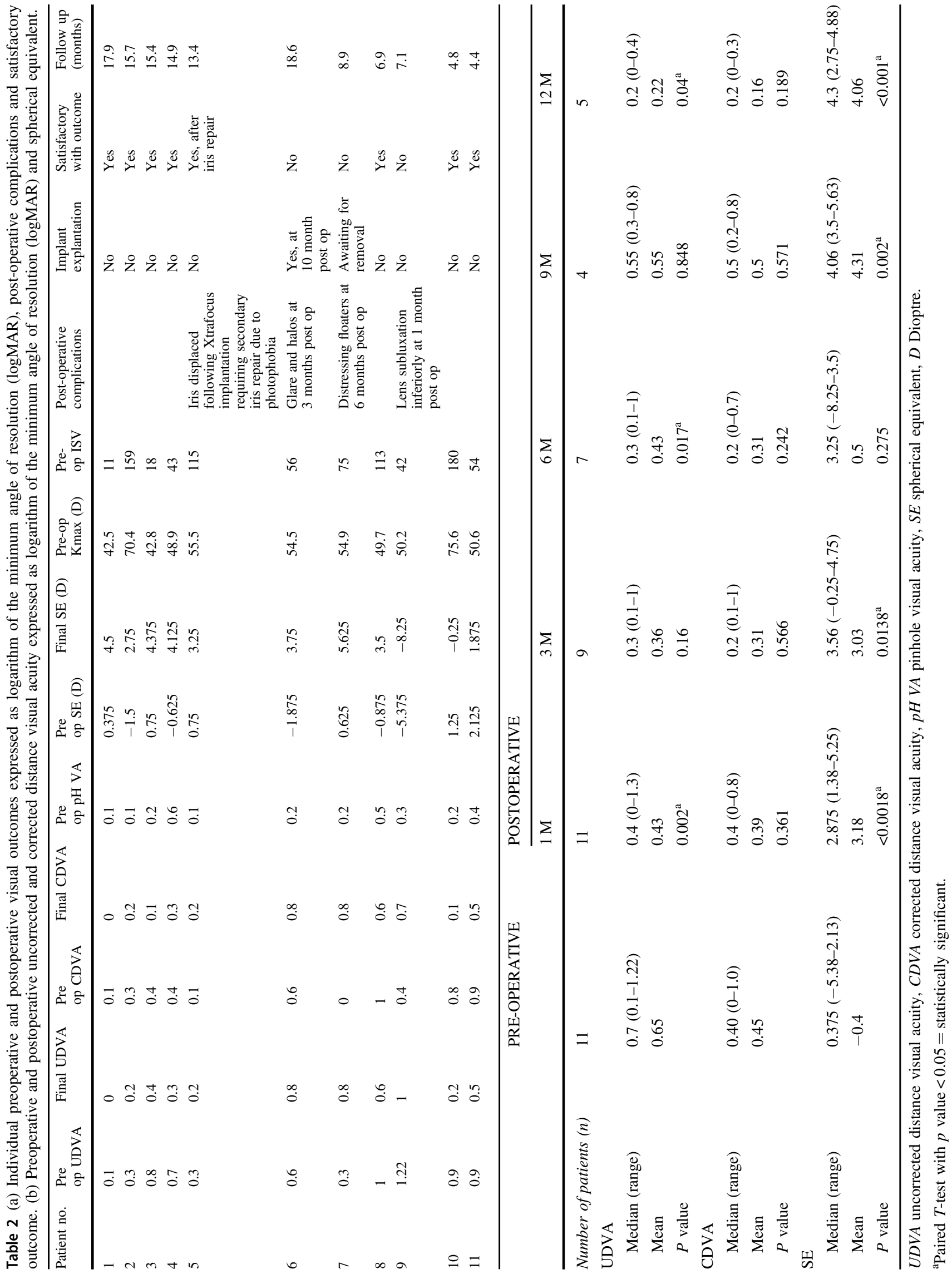

SPRINGER NATURE 
Trindade et al [7]. reported in their sulcus case series that one patient had a temporal decentration causing a dark crescent in the temporal field of vision, which resolved following surgical repositioning. In their larger series [10], decentration was reported in $8.3 \%$ patients when the pinhole implant was positioned in the capsular bag together with a primary IOL, though repositioning was not required in any of these patients. They reported that the pinhole device could be implanted safely in both sulcus or within the capsular bag with the later offering better centration $[13,14]$. Two of our patients had implant centration issue resulting in fluctuating vision, though we do not believe it has any relation to the pinhole device. One patient had a pupil displacement noted following the surgery. He previously had a toric Artisan iris clip IOL, which was removed, followed by cataract extraction with monofocal IOL placed in the bag. An XtraFocus ${ }^{\mathrm{TM}}$ was implanted subsequently, but the pupillary opening was not coincided with the pinhole aperture. At 3-months post pinhole implantation, an iris sphincterotomy was performed to uncover the pinhole aperture, with McCannel sutures to close the previously created peripheral iridectomy. This resulted in complete resolution of glare and vision improvement. Another patient developed subluxation of the device at 1-week despite an uneventful implantation. She chose to be observed with no repositioning at the time of writing. The patient had a routine DALK but developed Urrets-Zavalia syndrome resulting in severe glare; both cataract surgery and subsequent pinhole implantation were performed successfully with no complication reported. We believe the zonules might have been compromised preoperatively and the sulcus placement of the pinhole device might have further weakened the zonules leading to subluxation, though there was no intra-operative complication recorded during the pinhole implantation. Ultrasound biomicroscopy (UBM) is not routinely performed in our unit for a secondary piggyback lens implantation though it would have helped to access the lens stability in this patient pre-operatively.

Our study has few limitations: first this is a retrospective study with no comparable controls to validate our results; second, although we were able to show an immediate visual improvement following surgery, we were unable to demonstrate long-term visual stability due to a shorter follow-up period ( $<9$ months) in four of our patients. In fact, the recent Coronovirus disease (COVID-19) pandemic has limited our ability to review patients in clinic and collect more informative data. We were however able to conduct telephone consultation at their 9- and 12-month post-op review and all four patients reported visual stability. Third, we were unable to draw any conclusion to whom may benefit most from the pinhole device due to the small sample size. Future study with larger patient sample and longer follow-up period would provide more answers to patient selection and the long-term safety and efficacy of the pinhole device.

In conclusion, this is the first UK published series on the use of XtraFocus ${ }^{\mathrm{TM}}$ pinhole device in pseduophakic patients with irregular corneal astigmatism and iris trauma. Our results demonstrated satisfactory visual outcome in over $70 \%$ patients at a 1-year period. Visual disturbances such as distressing floaters or glare preclude its use in some patients resulting in implant explantation.

\section{Summary}

\section{What was known before}

- Secondary piggyback pinhole device (Xtrafocus TM) implantation is indicated for the treatment of irregular corneal astigmatism in pseudophakic patients. It has also been used in patients with combined irregular astigmatism and large iris defect.

\section{What this study adds}

- Our experience from a UK tertiary eye care centre has shown it to be effective in improving vision or reducing glare in these patients. Disturbing floaters and glare preclude its use in some resulting in implant explantation.

Author contributions $\mathrm{VH}$ was responsible for collecting and analysing data, interpreting results, writing the abstract, tables and the paper. ME and $\mathrm{SH}$ contributed to reviewing of the data, interpreting results and providing feedback on the paper. DL contributed to the design of the study, reviewing and providing feedback on the paper.

\section{Compliance with ethical standards}

Conflict of interest The authors declare no competing interests.

Publisher's note Springer Nature remains neutral with regard to jurisdictional claims in published maps and institutional affiliations.

\section{References}

1. Alió JL, Belda JI, Artola A, García-Lledó M, Osman A. Contact lens fitting to correct irregular astigmatism after corneal refractive surgery. J Cataract Refract Surg. 2002;28:1750-57.

2. Uçakhan ÖÖ, Yeşiltaş YS. Correction of Irregular Astigmatism With New-Generation Hybrid Contact Lenses. Eye Contact Lens. 2020;46:91-8. 
3. Rathi V, Mandathara P, Taneja M, Dumpati S, Sangwan V. Scleral lens for keratoconus: technology update. Clin Ophthalmol. 2015;9:2013-18.

4. Kwitko S, Cabral FP, de Araújo BS, Jung YP. Topography-guided ablation for the treatment of irregular astigmatism. J EuCornea. 2019;2:20-3.

5. Ferrara G, Torquetti L, Ferrara P, Merayo-Lloves J. Intrastromal corneal ring segments: visual outcomes from a large case series. Clin Exp Ophthalmol. 2012;40:433-9.

6. Tognon T, Campos M, Wengrzynovski JP, Barella KA, Pasqualotti A. de Brito Martins, et al. Indications and visual outcomes of intrastromal corneal ring segment implantation in a large patient series. Clinics. 2017;72:370-7.

7. Trindade CC, Trindade BC, Trindade FC, Werner L, Osher R, Santhiago MR. New pinhole sulcus implant for the correction of irregular corneal astigmatism. J Cataract Refract Surg. 2017;43:1297-1306.

8. Agarwal P, Navon SE. XtraFocus pinhole IOL (Morchers GMBH) a novel approach to tackle irregular astigmatism and large pupillary defects with a single step surgery. BMJ Case Rep. 2019;12: e228902.
9. Soeters N, Muijzer MB, Molenaar J, Godefrooij DA, Wisse RPL. Autorefraction Versus Manifest Refraction in Patients With Keratoconus. J Refract Surg. 2018;34:30-4.

10. Agarwal P, Navon SE, Subudhi P, Mithal N. Persistently poor vision in dim illumination after implantation of XtraFocus smallaperture IOL (Morcher). BMJ Case Rep. 2019;12:e232473.

11. Agarwal P, Navon SE. Persistent troublesome floaters necessitating the explantation of XtraFocus Pinhole IOL (Morcher). BMJ Case Rep. 2019;12:e229057.

12. Lam DS, Leung HY, Liu S, Radke N, Yuan Y, Lee VY. Two-Port Pars Plana Anterior and Central Core Vitrectomy (Lam Floaterectomy) in Combination With Phacoemulsification and Intraocular Lens Implantation Under Topical Anesthesia for Patients with Cataract and Significant Floaters: results of the First 50 Consecutive Cases. Asia Pac J Ophthalmol (Philos). 2017;6:33-6.

13. Trindade BC, Trindade FC, Werner L, Trindade CLC. Long-term safety of in-the-bag implantation of a supplementary intraocular pinhole. J Cataract Refract Surg. 2020;46:888-92.

14. Trindade BLC, Trindade FC, Trindade CLC. Intraocular pinhole implantation for irregular astigmatism after planned and unplanned posterior capsule opening during cataract surgery. J Cataract Refract Surg. 2019;45:372-7. 